Draft version December 4, 2018

Typeset using $\mathrm{LATEX}_{\mathrm{E}}$ preprint style in AASTeX61

\title{
SEARCHING A THOUSAND RADIO PULSARS FOR GAMMA-RAY EMISSION
}

\author{
D. A. Smith,${ }^{1}$ P. Bruel ${ }^{2}$ I. Cognard,${ }^{3,4}$ A. D. Cameron,${ }^{5,6}$ F. Camilo, ${ }^{7}$ S. Dai,${ }^{5}$ \\ L. Guillemot,${ }^{3,4}$ T. J. Johnson ${ }^{8}$ S. Johnston,${ }^{5}$ M. J. Keith,${ }^{9}$ M. Kerr, ${ }^{10}$ M. Kramer,${ }^{6,9}$ \\ A. G. Lyne, ${ }^{9}$ R. N. Manchester,${ }^{5}$ R. Shannon, ${ }^{11}$ C. Sobey,${ }^{12,13}$ B. W. Stappers,${ }^{9}$ And \\ P. Weltevrede ${ }^{9}$
}

${ }^{1}$ Centre d'Études Nucléaires de Bordeaux Gradignan, IN2P3/CNRS, Université Bordeaux 1, BP120, F-33175 Gradignan Cedex, France

${ }^{2}$ Laboratoire Leprince-Ringuet, École polytechnique, CNRS/IN2P3, F-91128 Palaiseau, France

${ }^{3}$ Laboratoire de Physique et Chimie de l'Environnement et de l'Espace - Université d'Orléans / CNRS, F-45071 Orléans Cedex 02, France

${ }^{4}$ Station de radioastronomie de Nançay, Observatoire de Paris, CNRS/INSU, F-18330 Nançay, France

${ }^{5}$ CSIRO Astronomy and Space Science, Australia Telescope National Facility, P.O. Box 76 Epping, NSW 1710, Australia

${ }^{6}$ Max-Planck-Institut für Radioastronomie, Auf dem Hügel 69, D-53121 Bonn, Germany

${ }^{7}$ Square Kilometre Array South Africa, Pinelands, 7405, South Africa

${ }^{8}$ College of Science, George Mason University, Fairfax, VA 22030, resident at Naval Research Laboratory, Washington, DC 20375, USA

${ }^{9}$ Jodrell Bank Centre for Astrophysics, School of Physics and Astronomy, The University of Manchester, M13 9PL, UK

${ }^{10}$ Space Science Division, Naval Research Laboratory, Washington, DC 20375-5352, USA

${ }^{11}$ ARC Centre of Excellence for Gravitational Wave Discovery, Centre for Astrophysics and Supercomputing, Swinburne University of Technology, Hawthorn, VIC 3122, Australia

${ }^{12}$ CSIRO Astronomy and Space Science, PO Box 1130, Bentley, WA 6102, Australia

${ }^{13}$ International Centre for Radio Astronomy Research - Curtin University, GPO Box U1987, Perth, WA 6845, Australia

\section{Accepted by ApJ}

\begin{abstract}
Identifying as many gamma-ray pulsars as possible in the Fermi Large Area Telescope (LAT) data helps test pulsar emission models by comparing predicted and observed properties for a large, varied sample with as little selection bias as possible. It also improves extrapolations from the observed population to estimate the contribution of unresolved pulsars to the diffuse gamma-ray emission. We use a recently developed method to determine the probability that a given gamma-ray photon comes from a known position in the sky, convolving the photon's energy with the LAT's energydependent point-spread-function (PSF), without the need for an accurate spatial and spectral model of the gamma-ray sky around the pulsar. The method is simple and fast and, importantly, provides probabilities, or weights, for gamma rays from pulsars too faint for phase-integrated detection. We applied the method to over a thousand pulsars for which we obtained rotation ephemerides from
\end{abstract}

smith@cenbg.in2p3.fr

Philippe.Bruel@llr.in2p3.fr 
radio observations, and discovered gamma-ray pulsations from 16 pulsars, 12 young and 4 recycled. PSR J2208+4056 has spindown power $\dot{E}=8 \times 10^{32} \mathrm{erg} \mathrm{s}^{-1}$, about three times lower than the previous observed gamma-ray emission "deathline". PSRs J2208+4056 and J1816-0755 have radio interpulses, constraining their geometry and perhaps enhancing their gamma-ray luminosity. We discuss whether the deathline is an artifact of selection bias due to the pulsar distance.

Keywords: gamma rays: observations - pulsars: individual (J0636+5129, J1731-4744, J1816-0755, J2208+4056) 


\section{INTRODUCTION}

The lowest photon fluxes among the 117 gamma-ray pulsars reported in the Second Fermi Large Area Telescope (LAT) Catalog of Gamma-ray Pulsars (Abdo et al. 2013, hereafter "2PC") were of order $F_{100}^{\min } \simeq 10^{-9}$ photons $\mathrm{cm}^{-2} \mathrm{~s}^{-1}$, integrated above $100 \mathrm{MeV}$. The LAT's effective area averaged over the energy range where pulsars are bright is $\sim 5000 \mathrm{~cm}^{2}$ (Atwood et al. 2009). Fermi's continuous all-sky survey covers every point on the sky with a roughly $\frac{1}{6}$ duty cycle. Combining, we find that $F_{100}^{\min }$ yields a modest average of 2 photons per month recorded by the LAT, with $>100 \%$ Poisson fluctuations in the monthly counting rate.

Detecting lower-flux pulsars gives access to a larger space volume, containing a rich variety of pulsars. Generally, luminosity scales with spindown power, $L_{\gamma} \propto \sqrt{\dot{E}}$ (see for example 2PC Fig. 9). However the dispersion around this trend spans over two orders of magnitude, presumably due to how the size, shape and density of the emitting region depend on the neutron star's spin and magnetic field configuration. Luminosity may thus be intrinsically low for pulsars with rare combinations of properties, or may appear low because only a shoulder of the gamma-ray beam sweeps across the Earth (Romani et al. 2011). "Variety" therefore includes pulsars probing the $\dot{E}=4 \pi^{2} I \dot{P} P^{-3}$ deathline for gamma-ray emission. $P$ is the period, $\dot{P}=\frac{d P}{d t}$, and we set the moment of inertia to $I=10^{45} \mathrm{~g} \mathrm{~cm}^{2}$.

Pulsars may be "faint", i.e. near the LAT's detection threshold, even for high count rates: background is intense for low Galactic latitudes and in confused regions. Broad pulse widths also degrade sensitivity (Hou et al. 2014). Detecting and characterizing faint pulsars helps ensure that emission models are confronted with an accurate snapshot of the true pulsar population. It also helps determine the pulsar luminosity function needed to quantify the contribution of unresolved pulsars to the diffuse emission, for example in the inner galaxy (Di Mauro et al. 2017).

We have been phase-folding LAT gamma-ray photons using hundreds of rotation ephemerides provided by the astronomers of the Pulsar Timing Consortium (Smith et al. 2008) since Fermi began routine observations on 2008 August 4 (MJD 54682). The Consortium provided ephemerides for $\sim 95 \%$ of known pulsars with spindown power $\dot{E}>10^{34} \mathrm{erg} \mathrm{s}^{-1}$, and about half of those known with lower $\dot{E}$. This led to the discovery of gamma pulsations from roughly half of LAT's over 240 gammaray pulsars ${ }^{1}$. The rest came from searches at the positions of pulsar-like unidentified LAT sources: a quarter of the total are radio-quiet pulsars found in blind gamma-ray periodicity searches (see Clark et al. 2017, and references therein), and nearly 90 are millisecond pulsars (MSPs) discovered via deep radio exposures (see e.g. Pleunis et al. 2017). But flagging a source as pulsar-like means it is bright enough in gamma rays to find its spectral shape and/or variability. The faint gamma-ray pulsars were all found by phase-folding with an ephemeris.

Weighting (Kerr 2011) is a key tool for these searches: the spatial and spectral map of the gamma rays around the pulsar's position, combined with the LAT's energy-dependent Point Spread Function (PSF), translates into the probability of a given photon being signal or background (see Section 2). What is new in the present work is that we applied the simplified weighting method of Bruel (2018) to discover even fainter objects, for which a phase-integrated source is often not even detectable.

In Section 2 we summarize the Bruel (2018) photon weighting method. The new photon weighting scheme is fast, making it useful for brighter sources as well. In Section 3 we use the method to

\footnotetext{
${ }^{1}$ https://confluence.slac.stanford.edu/display/GLAMCOG/Public+List+of+LAT-Detected+Gamma-Ray+Pulsars
} 
phase-fold 1269 pulsars, over 240 of which were known to pulse in gamma rays as this work was being completed. The large sample highlights the stability and robustness of our search method, in particular that the significance "noise floor" is safely below $4 \sigma$, lower than the $5 \sigma$ threshold used in previous work. Gamma-ray pulsations from 16 previously-known energetic radio pulsars are presented in Section 3. Folding the large sample also pushes the apparent gamma-ray emission "deathline" for pulsars to spindown power $\dot{E} \lesssim 8 \times 10^{32} \mathrm{erg} \mathrm{s}^{-1}$ (Section 4). We summarize in Section 5.

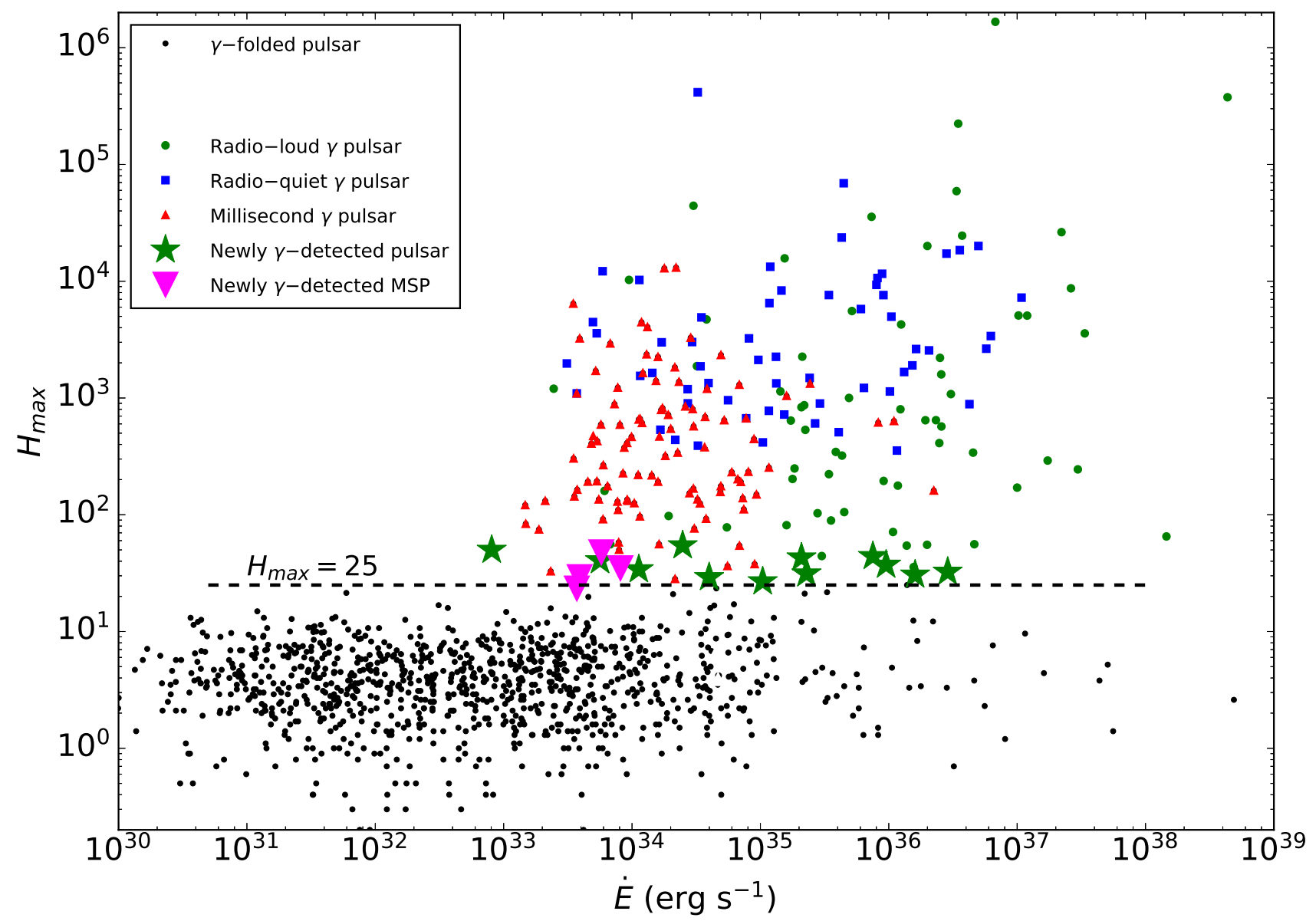

Figure 1. $H_{\max }$ (see Section 3) versus the spindown power $\dot{E}$ for 1269 pulsars, for 9.6 years of LAT data. Colored symbols indicate those known to pulse in LAT gamma rays. Small black dots show pulsars that were gamma-ray phase-folded, for which pulsations are not detected. The Shklovskii $\dot{E}$ correction for proper motion has not been applied here. The 16 gamma-ray pulsars discovered in the course of this work are highlighted.

\section{WEIGHTED PHOTON FOLDING, REVISITED}

A gamma ray crosses the vacuum of space for thousands of years without incident, to enter perhaps into the LAT. Recoil by the atoms therein ensures conservation of momentum, allowing pair production, $\gamma \rightarrow e^{+} e^{-}$. The electron and positron ionize the silicon strips along their path in the 
LAT's tracker, but undergo multiple Coulomb scattering, mainly in the tungsten foils. Coulomb scattering causes the fundamental limit on the angular resolution of the LAT at low energy, and determines its PSF. The spacing between the silicon strips limits the minimum PSF above $10 \mathrm{GeV}$. Smith \& Thompson (2009) provide further discussion.

At the end of the complex chain of LAT event reconstruction and selection, the LAT's energydependent PSF is modeled as a Moffat distribution (see Eq. 3, below) in $\Delta \theta$, the angular distance between a target direction (here, the pulsar's radio timing position) and a photon's sky direction $\vec{\theta}$. The distribution's width in degrees $(68 \%$ containment) is

$$
\sigma_{\mathrm{psf}}\left(E_{\gamma}\right)=\sqrt{p_{0}^{2}\left(E_{\gamma} / 100\right)^{-2 p_{1}}+p_{2}^{2}}
$$

where $E_{\gamma}$ is the gamma-ray photon energy in MeV. For the P305 Pass 8 data $^{2}$ used in this work (Atwood et al. 2013), the parameter values are $p_{0}=5.445, p_{1}=0.848$, and $p_{2}=0.084$.

To search for a pulsed gamma-ray signal, we select gamma-ray photons within $5^{\circ}$ of the pulsar position, approaching $\sigma_{\text {psf }}$ for our $E_{\gamma}>50 \mathrm{MeV}$ data sample. Their arrival times in the LAT are converted to a rotational phase of the neutron star using the parameters in the rotation ephemeris for that pulsar and the fermi plug-in (Ray et al. 2011) to Tempo2 (Hobbs et al. 2006). We evaluate pulsation significance for the photon list with the H-test (de Jager \& Büsching 2010). We stack (fold) the phases in histograms to examine and analyze the profile.

In the presence of background photons from nearby sources and diffuse emission, the signal-to-noise ratio can be optimized using different methods. The most effective to date is weighting (Kerr 2011). The weight $w\left(E_{\gamma}, \vec{\theta}\right)$ is the probability that a photon with energy $E_{\gamma}$ and apparent sky direction $\vec{\theta}$ comes from the pulsar rather than background. Kerr calculates the weights as $w=R_{p} /\left(R_{b}+R_{p}\right)$, where $R_{p}$ is the rate of gamma rays from the pulsar, and $R_{b}$ is the rate for gamma rays from all the background components. The Fermi Science Tool gtsrcprob implements the method. It takes as input a "source model" describing the spectra and positions of all nearby gamma-ray sources, created using the gtlike tool, as well as $\sigma_{\text {psf }}\left(E_{\gamma}\right)$ and $\vec{\theta}$, to calculate the expected rates and thus $w$. Kerr modified the H-test to include weights, summarized succintly in Section 3 of $2 \mathrm{PC}$. Weighting for the 117 pulsars in 2PC, and for nearly all LAT team pulsar studies since 2011, was done with gtsrcprob.

However, the gtsrcprob tool has two significant drawbacks. First, gtlike requires high detection significance to allow gtsrcprob weights to be meaningful. The analysis can be complex for faint sources in highly confused regions, and may fail for faint sources where pulsations are nevertheless clearly detectable. Second, gtlike, and the tools gtdiffrsp and gtexposure that its use requires, impose much larger data files and longer computation times than needed for the simple weighting method.

\subsection{Simplified Weighting}

Bruel (2018) provides a simple formula that approximates the weights remarkably well, ${ }^{3}$

$$
w\left(E_{\gamma}, \Delta \theta\right)=g\left(E_{\gamma}, \Delta \theta\right) \exp \left(-2 \log ^{2}\left(E_{\gamma} / E_{\text {ref }}\right)\right),
$$

with one free parameter for the energy scale, $\mu_{w}=\log _{10}\left(E_{\text {ref }} / 1 \mathrm{MeV}\right)$, discussed below. The geometrical factor $g$ uses a Moffat distribution (Moffat 1969) ${ }^{4}$ to describe the angular distribution of the

\footnotetext{
2 https://fermi.gsfc.nasa.gov/ssc/data/

3 A python script to calculate the weights is at https://fermi.gsfc.nasa.gov/ssc/data/analysis/user, and also at https://github.com/nanograv/PINT/blob/master/pint/fermi_toas.py .

${ }^{4}$ Mis-attributed to King (1962) in Read et al. (2011), corrected in the LAT analysis documentation.
} 


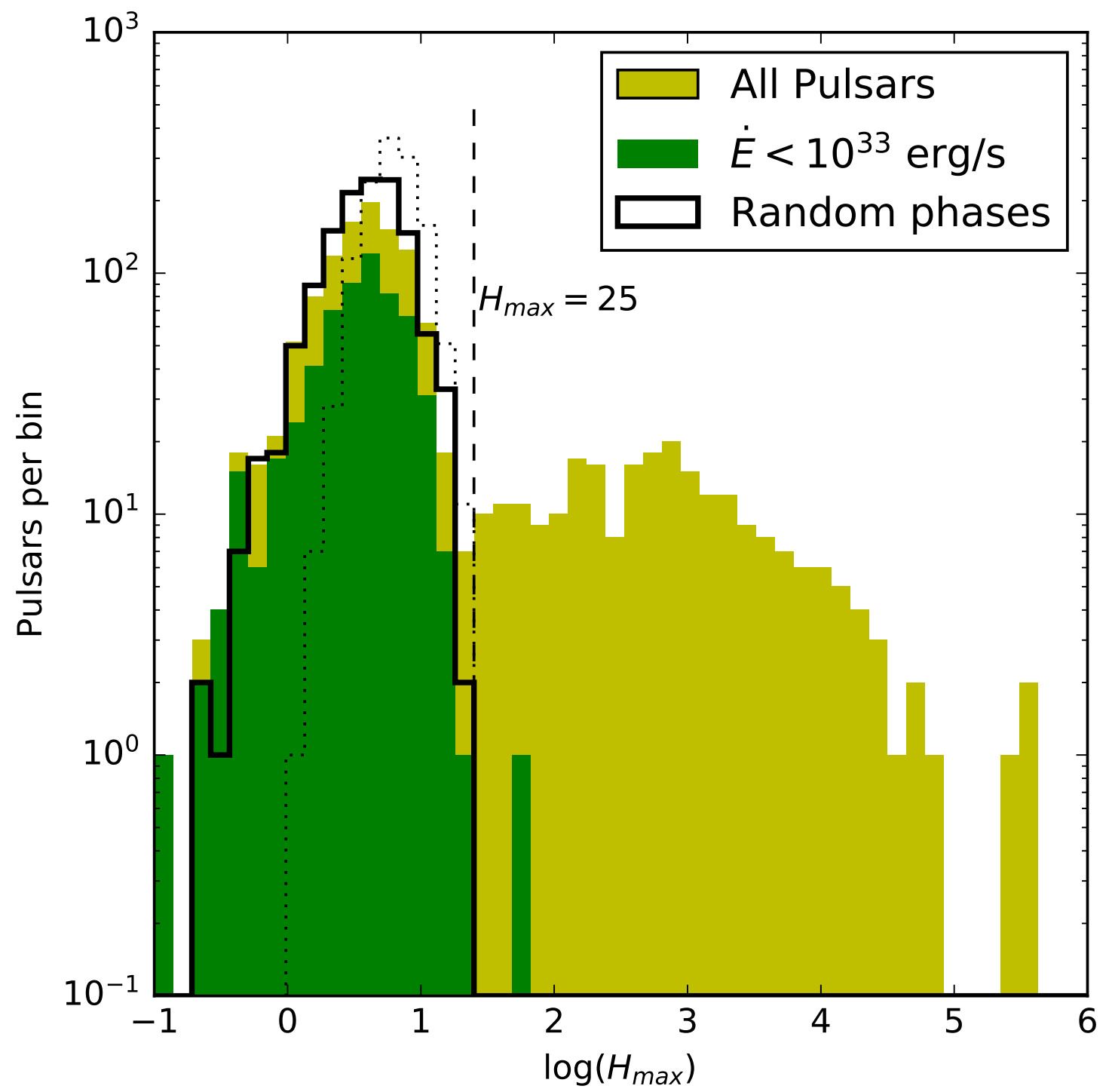

Figure 2. Histograms of the $H_{\max }$ values shown in Figure 1, and of the maximum H-test values obtained for 3 (solid line) and 8 (dotted line) sets of random phases generated for each pulsar's photon list (see Section $3)$.

gamma-ray photons emanating from a point source,

$$
g\left(E_{\gamma}, \Delta \theta\right)=\left(1+\frac{9 \Delta \theta^{2}}{4 \sigma_{\mathrm{psf}}^{2}\left(E_{\gamma}\right)}\right)^{-2}
$$

The Moffat distribution resembles a Gaussian but with broader tails.

The exponential factor was obtained by studying weight distributions computed under the simple assumptions that the pulsar rate is much lower than the background rate, and that the background 
is uniform. Bruel (2018) explores the validity of these assumptions. Pulsar spectra are of the form

$$
\frac{\mathrm{d} N}{\mathrm{~d} E_{\gamma}}=K\left(\frac{E_{\gamma}}{1 \mathrm{GeV}}\right)^{-\Gamma} \exp \left(-\frac{E_{\gamma}}{E_{\text {cut }}}\right)
$$

that is, hard spectra (typically $0.5<\Gamma<2.0$ ) with sharp cut-offs $\left(0.6<E_{\text {cut }}<6 \mathrm{GeV}\right.$, see $2 \mathrm{PC}$ ). $\mathrm{K}$ determines the flux. In the energy range germane to pulsars, the spectrum of the diffuse background is a steeper power law. In these conditions, the pulsar weights versus $\log E_{\gamma}$ are well-approximated by Gaussians with width 0.5 , relatively independent of the pulsar's spectrum. What changes from one pulsar to another is the reference energy $E_{\text {ref }}=10^{\mu_{w}}$ for which the pulsar's weight distribution peaks. For a faint pulsar with a soft spectrum far off the Galactic plane (hence, with low background), $E_{\text {ref }}$ can be below $1 \mathrm{GeV}\left(\mu_{w}=3\right)$. A pulsar with a hard spectrum in a highly confused region can have a distribution peaking above $10 \mathrm{GeV}\left(\mu_{w}=4\right)$.

\subsection{Choosing $\mu_{w}$}

For a detectable gamma-ray pulsar, H-test versus $\mu_{w}$ follows a bell-like curve. To find $E_{\text {ref }}$ that maximizes pulsation significance, Bruel (2018) describes a simple procedure to find the curve's peak with the fewest trials. Here, we proceeded differently. After phase-folding many gamma-ray pulsars, we saw that for most, $\mu_{w}=3.6$ yields a significant signal. The values of $\mu_{w}=3.2$ and 4.0 work for the rest. After detection, the above fitting procedure tunes $\mu_{w}$ to optimize the $\mathrm{H}$-test significance. Often, to confirm a pulsar discovery, radio and/or gamma-ray timing (Ray et al. 2011) allows the gamma rays to be phase-folded over a longer epoch, and the pulsed significance becomes high enough that trials penalities incurred by optimizing $\mu_{w}$ do not compromise confidence in the detection.

PSR B1509-58 has long been notorious for having by far the softest spectrum of any gamma-ray pulsar, with a spectral cut-off below $50 \mathrm{MeV}$ (Abdo et al. 2010). It is so bright that it is easily detected for a broad range of $\mu_{w}$ values, but its highest pulsed significance is with the low value of $\mu_{w}=2.3$. Kuiper \& Hermsen (2015) argue that PSR B1509-58 is one of a class of pulsars with spectra peaking in the $\mathrm{MeV}$ range. To enhance our sensitivity to faint soft-spectra pulsars, we repeated our search with $\mu_{w}=2.3$. No new pulsars were found. We note that the gamma-ray pulsar detections described below were initially made using photons with $E_{\gamma}>100 \mathrm{MeV}$ and $\Delta \theta<2^{\circ}$. The data files are $\approx 6$ times smaller than the $E_{\gamma}>50 \mathrm{MeV}$ data sets, easing calculations, albeit with less sensitivity to pulsars having spectra like PSR B1509-58's.

\subsection{Rotation Ephemerides}

Phase-folding gamma rays requires phase-connected rotation ephemerides accurate during the Fermi epoch. Ephemeris timing residuals with root-mean-squares smaller than $P / 50$ provide adequate accuracy for all gamma-ray pulsars known so far. Routine observations of many pulsars stop within a few years of their discovery, however, and such ephemerides often do not exist. For this reason, to prepare for the Fermi mission, radio and X-ray astronomers organized to provide ephemerides for the 230 pulsars with spindown power $\dot{E}>10^{34} \mathrm{erg} \mathrm{s}^{-1}$ that were known at the time (Smith et al. 2008). Astronomers from Parkes Observatory in Australia (Weltevrede et al. 2010), from Jodrell Bank Observatory in England (Hobbs et al. 2004b), and from Nançay in France (Cognard et al. 2011) continue to provide timing data not only for most of the original list, but also for high $\dot{E}$ pulsars discovered subsequently and, key to this work, for a thousand pulsars with lower spindown power. Astronomers from Effelsberg (Germany), Green Bank (USA), Arecibo (Puerto Rico), the 
Giant Metrewave Radio Telescope (India), Westerbork (Netherlands), and other radio telescopes, as well as X-ray astronomers also help extract as many pulsed signals as possible from the LAT data by providing rotation ephemerides to the LAT team. In particular, the Green Bank Northern Celestial Cap (GBNCC) pulsar survey (Lynch et al. 2018, and references therein) and the High Time Resolution Universe (HTRU) pulsar survey (Ng et al. 2015; Cameron et al. 2018, Cameron et al. in prep) provided dozens of rotation ephemerides. Half of the $\sim 2800$ known radio pulsars ${ }^{5}$ (Manchester et al. 2005) have not been explored for gamma-ray pulsations. Attempts to use older rotation parameters to seed a focused periodicity search engender so many trials that only highsignificance detections would be compelling, and are beyond the scope of this work.

We exclude the $\sim 170$ known pulsars in globular clusters from the results shown here: their unknown orbital acceleration within the cluster generally skews their observed $\dot{E}$ values relative to their true spindown powers, diminishing their usefulness for this study. We have however gamma ray phasefolded 53 for which we could obtain reliable ephemerides, using the methods described here. Beyond the re-detection of the two reported in $2 \mathrm{PC}$, no new discoveries were made.

"Ephemeris validity" strictly speaking is the interval between the first and last time-of-arrival measurements used to determine the rotation spindown parameters. However, for many pulsars spindown is stable and the predicted number of neutron star turns is accurate for months, even years, before and/or after that interval. Conversely, young high $\dot{E}$ pulsars can have erratic spindown fluctuations ("timing noise") requiring rotation models to use many fitted parameters. MSPs can too, although the fluctuations are generally smaller (Shannon \& Cordes 2010). Binary MSPs can undergo erratic orbital perturbations when the pulsar wind ablates the companion, as for example for PSR J1048+2339 (Deneva et al. 2016). Models for such pulsars predict the number of rotations, and thus phase, poorly outside the validity interval.

\section{SEARCHING A THOUSAND PULSARS FOR GAMMA RAYS}

In practice, we define $H_{\max }$ as the maximum H-test value for 6 trials: the 3 values of $\mu_{w}=3.2,3.6$ and 4.0 described above, applied to two data samples. One sample uses 9.6 years of LAT data, from the beginning of the Fermi mission, MJD 54682, until MJD 58182. The other uses LAT data only during the ephemeris validity period. Many pulsars rise to higher significance followed by a rollover at intermediate dates, but restricting to two epoch durations keeps the number of trials small and well-defined.

Figure 1 shows $H_{\max }$ versus $\dot{E}$ for 1269 pulsars, and Figure 2 shows a histogram of the same $H_{\max }$ values. A "noise floor" appears. We choose $H_{\max }>25$ (single trial weighted H-test significance of $4.1 \sigma$ ) as our threshold for detection of gamma-ray pulsations. With this threshold, we expect less than one false detection for our 1269 pulsar sample. We discuss the 16 gamma-ray pulsars thus discovered below.

Figure 2 also shows the distribution of $\mathrm{H}$-test values obtained for the same pulsar sample, where the photon phases were replaced with uniform random values between 0 and 1 , using $\mu_{w}=3.6$. We did this 3 times (solid histogram) and 8 times (dotted histogram) for each pulsar and kept the maximum value, as in the procedure for $H_{\max }$. The 3-trial histogram matches that of the undetected pulsars. Three trials suffice because the $\mu_{w}=2.3$ trials are not included in Figures 1 and 2, but also because counting both data sets is redundant: the "all data" and "ephemeris validity" H-test values are in

${ }^{5}$ See the ATNF Pulsar Catalog, http://www.atnf.csiro.au/research/pulsar/psrcat 

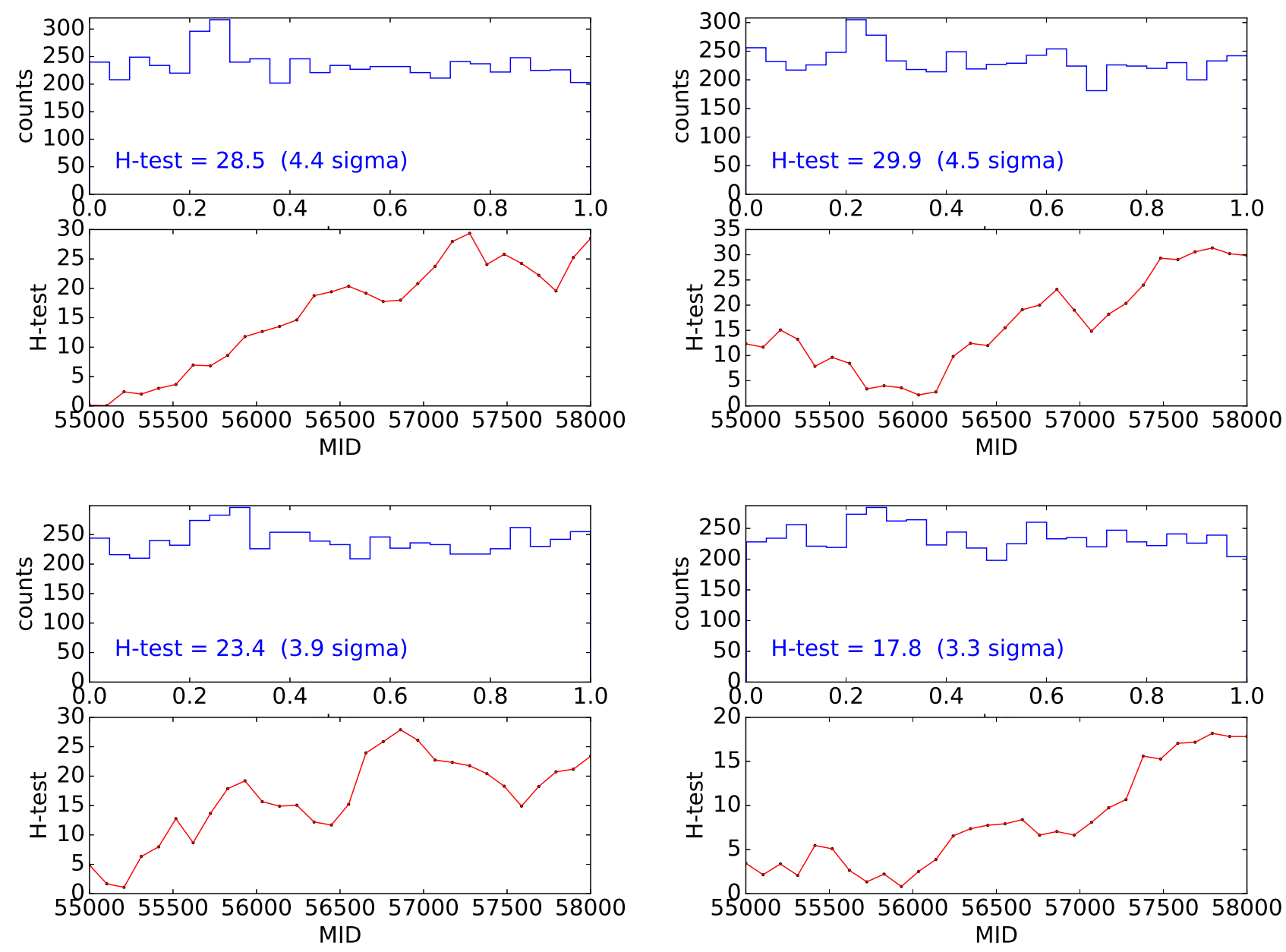

Figure 3. Four simulated LAT 8.2-year (3000 day) missions for a single pulsar with flux $\sim 1 \times 10^{-9}$ photons $\mathrm{cm}^{-2} \mathrm{~s}^{-1}$ and a typical low background rate. Top frames: phase-histogram integrated over the mission. Bottom frames: Integrated significance as the data accumulates over time (MJD = Modified Julian Day). The pulsar is detected when H-test $>25$. For these parameters, half of the trials yield H-test $<15$. Large fluctuations in cumulated significance result from Poisson fluctuations in the low photon arrival rate.

fact highly correlated. Two-thirds (one-third) of our ephemerides cover over half (over 90\%) of the mission. Ephemeris validity is $<500$ days for only $5 \%$ of the sample. Eight trials adds the fourth scan, with $\mu_{w}=2.3$, for the two data samples, ignoring correlations between the samples. $H_{\max }>25$ never occurs in the random histograms.

A simple Monte Carlo calculation, illustrated in Figure 3, gives insight. Four simulated examples of significance growth versus time are shown. For the few photons per month signal rates evoked in the Introduction for pulsars near detection threshold, Poisson fluctuations can cause irregular growth, sometimes wildly so. We see such fluctuations in the data, and speculated that they could be due to changes in flux, instrument exposure, or ephemeris instability. In fact, Poisson fluctuations dominate. For the simulated signal and background rates in Figure $3, F_{100}^{\min } \simeq 10^{-9}$ photons $\mathrm{cm}^{-2}$ $\mathrm{s}^{-1}$, we simulated the Fermi mission 1000 times. The mean single-trial significance is $\sim 4 \sigma$, meaning that the pulsar is not detected half the time. The full width at half maximum of the significance 
distribution extends from $2.5 \sigma$ to $5 \sigma$. We also used the simulation to understand how many years it takes to detect a pulsar having a given flux, duty cycle, and background environment. Statistical fluctuations smear the answer. Near threshold, the fluctuations are Poissonian and can be so large that $H_{\max }>25$ can occur a full year earlier or later than the average duration.

\subsection{Discoveries of Gamma Rays from Radio Pulsars}

Table 1 lists 16 gamma-ray pulsars discovered using the above method. Twelve are young to middleaged, and four are MSPs. Figures 4, 5 and 6 show their phase-aligned $>50 \mathrm{MeV}$ gamma-ray and radio profiles, as well as the evolution of their H-test (pulsed Test Significance) since the start of the Fermi mission.

\subsubsection{Non-recycled Pulsars}

The parameter space occupied by gamma-ray pulsars continues to fill in and expand. PSR J2208+4056 has $\dot{E}=8 \times 10^{32} \mathrm{erg} \mathrm{s}^{-1}$, three times lower than the previous lowest spindown power for young gamma-ray pulsars. PSR J0729-1836 is now one of nine young pulsars with $\dot{E}<10^{34} \mathrm{erg}$ $\mathrm{s}^{-1}$. At the other end of the range, PSRs J1913+1011 and J1928+1746 both have $\dot{E}>10^{36} \mathrm{erg} \mathrm{s}^{-1}$.

PSR J1731-4744 is the longest spin-period gamma-ray pulsar now known, with $P=830 \mathrm{~ms}$. Slow pulsars (PSR J0729-1836 has $P=510 \mathrm{~ms}$ ) attain $\dot{E} \simeq 10^{34} \mathrm{erg} \mathrm{s}^{-1}$ because their surface magnetic fields are high, $B_{S} \gtrsim 10^{13} \mathrm{G}$. In contrast, PSR J1913+1011 is nearly as fast as the Crab but with $B_{S}$ an order of magnitude smaller.

In the LAT 8-year preliminary source list ${ }^{2}$, FL8Y, only the six pulsars flagged in Table 1 lie within a source's $95 \%$ confidence ellipse. The rest are undetected, using a procedure similar to that of 3FGL (Acero et al. 2015), improved in preparation for 4FGL, the upcoming $4^{\text {th }}$ Fermi LAT catalog. Weighted folding improves signal-to-noise roughly as the inverse of the pulse width $\delta \phi$, compared to a phase-integrated detection. Seven of the twelve young pulsars have Galactic latitude $|b|<0^{\circ} .6$ (and thus very high background levels), and all but one of these (PSR J1853-0004) have narrow gamma-ray pulses, with $\delta \phi<0.3$ in phase.

The highest background levels are towards the Galactic center. The detection of PSR J1757-2421, only $5^{\circ} .3$ away, is aided by its narrow pulse, $\delta \phi<0.1$. PSR J1841-0524, with high $\dot{E}$ and intermediate distance, presumably needed over nine years of LAT data to be seen because its overlap with the bright pulsar wind nebula HESS J1841-055 raises its background (Acero et al. 2013). Only $0^{\circ} .9$ separate high $\dot{E}$ PSRs J1925+1720 and J1928+1746, raising each's background marginally compared to the already high level of this busy region in the Galactic plane, compounding detection difficulty.

Finally, PSR J1932+2220 is the most distant of the new pulsars. The NE2001 (Cordes \& Lazio 2002) and the YMW16 (Yao et al. 2017) Dispersion Measure (DM) models place it at 7.5 and $8 \mathrm{kpc}$ from Earth, respectively, whereas its distance derived from HI absorption is $10.9_{-0.8}^{+0.3} \mathrm{kpc}$ (Verbiest et al. 2012). The others pulsars lie in the range of $\sim 1$ to $\sim 6 \mathrm{kpc}$, like the bulk of the 2PC pulsars. PSR J1932+2220's "faintness" is thus a combination of low flux (medium $\dot{E}$ at large distance) and the background level just off the plane.

\subsubsection{Millisecond Pulsars}

Three of the four new MSPs are "faint" simply due to low gamma-ray fluxes: neither the intense background at low latitudes nor broad peaks made them hard to detect. Three have distances $d<2$ kpc, as derived from their DM values. The only one in the FL8Y list, PSR J1125-6014, is also the 

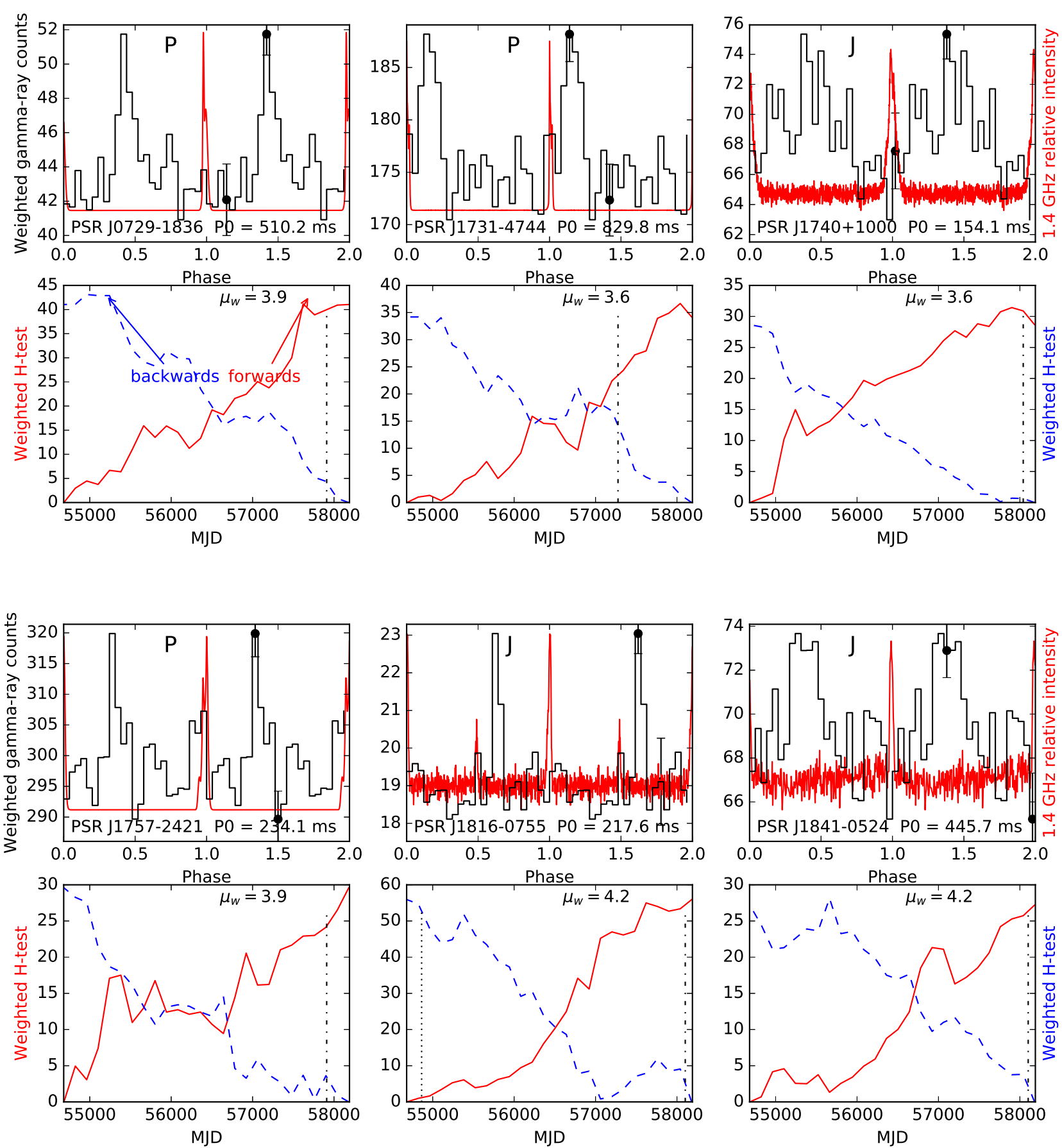

Figure 4. Six young and middle-aged pulsars newly discovered in gamma rays. Top: Weighted phase histograms of the $>50 \mathrm{MeV}$ gamma rays. The largest and smallest statistical uncertainties are shown. The overlaid $1.4 \mathrm{GHz}$ radio profiles are phase-aligned, with the letter indicating the radio telescope that recorded the profile: N, Nançay; P, Parkes ; J, Jodrell Bank, see the references in Table 1. A full rotation is shown twice, with 25 phase bins. Bottom: H-test significance cumulated starting at both the beginning (solid curve) and at the end (dashed curve) of the dataset. The vertical dotted (dot-dash) line indicates the start (finish) of the ephemeris validity. The weighting parameter $\mu_{w}$ used for each pulsar is shown. 

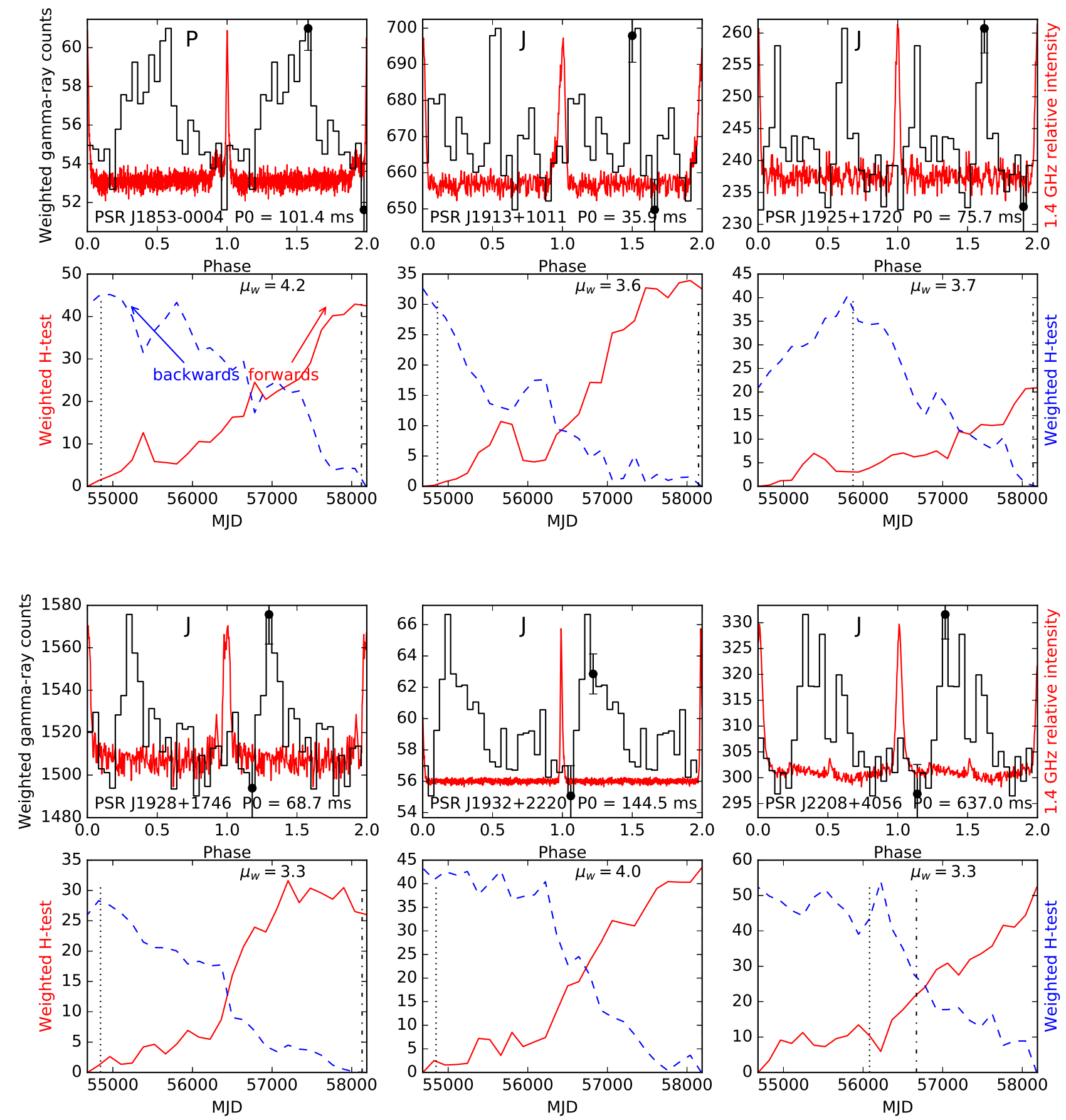

Figure 5. Six young pulsars newly discovered in gamma rays, as in Figure 4. For PSR J1925+1720 the phase histogram integrates data only after the beginning of the ephemeris validity. For the other pulsars, all data from MJD 54682 until MJD 58182 is used.

only one deep in the plane, with $b=0^{\circ} .9$, with five other gamma-ray pulsars less than $2^{\circ}$ away. It also has the narrowest pulse, facilitating detection. In Figure 6 its signal is contained in a single phase bin of width $\delta \phi=1 / 50=0.02$ in phase, so its pulse is $<53 \mu$ s wide. 
Barr et al. (2017) used timing of the binary orbit and radio polarization measurements to characterize PSR J1946+3417. They argue that the more intense radio peak (see Figure 6) could come from a polar cap, while the smaller one would come from the outer magnetosphere. They determined the pulsar's geometry to be favorable for gamma-ray viewing from Earth, and attributed its nondetection by the LAT to a large distance and low spindown power. Their reasoning overlaps that invoked for young radio interpulse pulsars in Section 4.1. PSR J1946+3417 is shown by the lowest large triangle in Figure 7, discussed below.

Having now detected it in gamma rays, we consider Barr et al's prediction that if indeed radio emission comes from an outer gap (OG), the gamma-ray emission would be aligned in phase with the second radio peak. The gamma-ray profile for PSR J1946+3417 in Figure 6 shows two peaks separated by 0.25 in phase. The radio peak they attribute to the $\mathrm{OG}$ is between them, on the inner shoulder of the trailing gamma peak, consistent with their prediction. The uncertainty shown is derived from the difference between the DM value in our ephemeris and that published by Barr et al. (2017). A wealth of accurate observational information now exists for this pulsar, making it an interesting candidate for detailed modeling.

For any pulsar, requiring gamma-ray efficiency $\eta=L_{\gamma} / \dot{E}<100 \%$ reveals its maximum possible distance, for a given assumption about its beaming fraction $f_{\Omega}$, because the luminosity $L_{\gamma}=4 \pi d^{2} f_{\Omega} G_{100}$ depends on the distance and on the energy flux integrated above $100 \mathrm{MeV}, G_{100}$. For MSPs, the Shklovskii effect makes the $\eta<100 \%$ constraint even stronger, because the intrinsic (corrected) spindown power $\dot{E}^{\text {int }}$ is also sensitive to distance, as illustrated in 2PC Figure 11 . We now explore the plausible distances of the four MSPs, and hence their possible $\dot{E}^{\text {int }}$ values.

The Doppler correction to $\dot{P}$ depends on the system's proper motion transverse to the line of sight, $\mu$ (Shklovskii 1970), and the acceleration due to its and the Sun's locations the Galaxy. From the observed spindown $\dot{P}$, the intrinsic value is $\dot{P}^{\text {int }}=\dot{P}-\dot{P}^{\text {shk }}-\dot{P}^{\text {gal }}$, with

$$
\dot{P}^{\text {shk }}=\frac{1}{c} \mu^{2} d P=k\left(\frac{\mu}{\text { mas yr }^{-1}}\right)^{2}\left(\frac{d}{\mathrm{kpc}}\right)\left(\frac{P}{\mathrm{~s}}\right)
$$

and

$$
\dot{P}^{\text {gal }}=\frac{1}{c} \mathbf{n}_{10} \cdot\left(\mathbf{a}_{1}-\mathbf{a}_{0}\right) P
$$

where $k=2.43 \times 10^{-21}$. The Galactic potential model of Carlberg \& Innanen (1987) and Kuijken \& Gilmore (1989) provides the accelerations $\mathbf{a}_{1}$ of the pulsar and $\mathbf{a}_{0}$ of the Sun. Here, $\mathbf{n}_{10}$ is the unit vector in the direction from the solar system to the pulsar.

For PSR J0636+5129, the two terms nearly cancel and the Shklovskii correction is small, $<1 \%$, as are the uncertainties, owing to a timing parallax distance measurement and a small, precise $\mu$ value (Stovall et al. 2014). PSR J1327-0755, in contrast, lies on a line-of-sight where converting the DM to distance is highly uncertain (see Table 1 ) and its nominally large $\mu$ value has large uncertainties. We therefore do not correct $\dot{P}$ for this MSP.

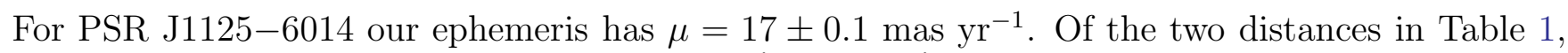
the closer one, $d=1 \mathrm{kpc}$, gives a correction of $\dot{E}^{\text {int }}=0.6 \dot{E}$. The $3 \mathrm{FGL}$ value of $G_{100}$ (Acero et al. 2015) and $f_{\Omega}=1$ then give a typical value of $\eta=30 \%$. The farther distance implies small $f_{\Omega}$ to reach that range of $\eta$ values.

Since PSR J1946+3417 is undetected in FL8Y, we have no $G_{100}$ value, and thus no $\eta<100 \%$ distance constraint. However, the "heuristic" energy flux $G_{h}=\sqrt{\dot{E}} /\left(4 \pi d^{2}\right)$ can also flag suspicious 
distances. In Figure 7, $G_{h}$ versus $\dot{E}$ for our twelve hundred pulsars, MSP J1946+3417 is well below any other gamma-ray pulsar, implying that the two DM distances in Table 1 are over-estimated. To raise PSR J1946+3417 to $G_{h}=8 \times 10^{14}\left(\mathrm{erg} \mathrm{s}^{-1}\right)^{1 / 2} \mathrm{kpc}^{-2}$ requires $d=2.5 \mathrm{kpc}$, two or three times closer than the DM distances.

The Shklovskii correction lowers $G_{h}$ for PSR J1946+3417 even more. Using $\mu=8.64 \pm 0.25$ mas $\mathrm{yr}^{-1}$ from Barr et al. (2017), for $d=7 \mathrm{kpc}$, observed $\dot{E}=3.8 \times 10^{33} \mathrm{erg} \mathrm{s}^{-1}$ corrects to a recordbreaking $\dot{E}^{\text {int }}=0.83 \times 10^{33} \mathrm{erg} \mathrm{s}^{-1}$. For $d=5.2 \mathrm{kpc}$, the corrected value is as small as any seen for a gamma-ray MSP, $\dot{E}^{\text {int }}=1.8 \times 10^{33} \mathrm{erg} \mathrm{s}^{-1}$, while $d=2.5 \mathrm{kpc}$ suggested by Figure 7 gives a more typical value of $\dot{E}^{\text {int }}=2.8 \times 10^{33} \mathrm{erg} \mathrm{s}^{-1}$. We add the $d=2.5 \mathrm{kpc}$ to Table 1 to underline that the gamma ray detection favors a closer distance. Finally, Barr et al. (2017) determined this pulsar to have $1.828 \pm 0.022$ solar masses, making $I$, and thus $\dot{E}, 30 \%$ larger than obtained using the usual Chandrasekhar mass for the neutron star. This shifts how a gamma-ray flux constrains this pulsar's distance.

\subsection{The Birthplace of PSR J1731-4744}

Discovered at the dawn of the pulsar era (Large et al. 1968), slowly spinning PSR J1731-4744 has a high magnetic field and thus a young characteristic age of $80 \mathrm{kyr}$. Shternin et al. (2017) compiled observations going back to 1971 and obtain a large proper motion with a small uncertainty, $146 \pm 12$ mas $\mathrm{yr}^{-1}$. We were unable to confirm their result with our timing data. Nevertheless, they argued that at the DM distance of 5.6 (2.8) kpc from the YMW16 (NE2001) model, their proper motion implies an exceptionally fast space velocity. They propose instead that PSR J1731-4744 was born in the supernova of which RCW 114 is the remnant (also known as SNR G343.0-6.0). The SNR distance is between 0.5 and $1 \mathrm{kpc}$. For $0.7 \mathrm{kpc}$, they find a more typical transverse speed of $v_{\perp} \simeq 500$ $\mathrm{km} \mathrm{s}^{-1}$. An unmodeled electron excess along the line-of-sight would explain an over-estimated DM distance. Their Figure 2 shows that the current pulsar position extrapolates back to the heart of RCW 114 over the system's age.

The gamma-ray detection of PSR J1731-4744 provides an independent constraint. The efficiency of $\eta=11 \%$ for $d=1 \mathrm{kpc}$ is typical for $2 \mathrm{PC}$ pulsars (for which the spread in $\eta$ is large). We find $\eta>100 \%$ for $d>3 \mathrm{kpc}$. In Figure 7, PSR J1731-4744 corresponds to the lowest star, for the YMW16 distance, even lower than B0540-69 in the LMC seen at far right.

SNR G343.0-6.0 is not detected in the Fermi LAT catalog of supernova remnants (Acero et al. 2016), nor in the more recent search for extended Galactic sources (Ackermann et al. 2017). Nevertheless, the gamma rays from the LAT source could be shared between the pulsar and the SNR. An extreme hypothesis that only a third of $G_{100}$ is due to the pulsar implies $\eta>100 \%$ for $d>5.2$ kpc. Comparing the on- and off-pulse spectral shapes may clarify the pulsar's contribution to the flux, although the source's faintness will limit the information that can be extracted. Smaller $f_{\Omega}$ would also enable a larger distance. With these caveats, we conclude that the gamma-ray detection supports the association of PSR J1731-4744 with RCW 114, and we adopt the nearer distance in Table 1.

\section{A GAMMA-RAY EMISSION DEATHLINE}

Striking in Figure 1 is a minimum spindown power $\dot{E}$ for gamma-ray pulsars. Most with $\dot{E}<10^{34}$ $\mathrm{erg} \mathrm{s}^{-1}$ are MSPs. The discovery of gamma-ray pulsations from PSR J2208+4056 establishes that 
Table 1. Twelve young (top) and four recycled (bottom) radio pulsars for which we see gamma-ray pulsations. The "Ref." column indicates the discovery paper. Distances are taken from the ATNF pulsar catalog. The first is derived from the dispersion measure (DM) using the YMW16 model (Yao et al. 2017) except for J1932+2220, which uses the kinematic distance of Frail \& Weisberg (1990), and for J0636+5129, for which Stovall et al. (2014) measured timing parallax. The second distance comes from the DM using NE2001 (Cordes \& Lazio 2002). The additional distance for J1731-4744 is from Shternin et al. (2017). The additional distance for J1946+3417 comes from the discussion of Figure 7, see text. The rotation ephemeris used in this work (last column) was obtained from radio timing with: N (Nançay Radio Telescope) Cognard et al. (2011) ; P (Parkes Radio Telescope) Weltevrede et al. (2010) ; J (Jodrell Bank Observatory) Hobbs et al. (2004b); E (Effelsberg) Barr et al. (2017) ; G (Green Bank Telescope) Kaplan et al. (2005). The observatories in parentheses also provided timing observations. The MSPs with $\dot{E}$ uncertainties are Shklovskii-corrected (see text). The 6 pulsars with a $\dagger$ are co-located with LAT 8-year sources.

\begin{tabular}{|c|c|c|c|c|c|c|c|c|c|}
\hline PSR & Ref. $^{\mathrm{a}}$ & $\begin{array}{r}l \\
\left({ }^{\circ}\right) \\
\end{array}$ & $\begin{array}{r}b \\
\left({ }^{\circ}\right) \\
\end{array}$ & $\begin{array}{r}P \\
(\mathrm{~ms}) \\
\end{array}$ & $\begin{array}{c}\text { Distance } \\
(\mathrm{kpc})\end{array}$ & $\begin{array}{r}\dot{E} / 10^{34} \\
\left(\operatorname{erg~s}^{-1}\right) \\
\end{array}$ & $\begin{array}{r}S_{1400} \\
(\mathrm{mJy}) \\
\end{array}$ & $\begin{array}{l}\text { H-test } \\
\text { signif. }\end{array}$ & Timing \\
\hline J0729-1836 & $\mathrm{mlt}+78$ & 233.76 & -0.34 & 510.2 & $2.4 / 2.9$ & 0.56 & 1.4 & 41 & $\mathrm{P}(\mathrm{J})$ \\
\hline $\mathrm{J} 1731-4744 \dagger$ & $\operatorname{lvw68}$ & 342.56 & -7.67 & 829.8 & $5.5 / 2.8(0.7)$ & 1.13 & 12.0 & 34 & $\mathrm{P}$ \\
\hline $\mathrm{J} 1740+1000 \dagger$ & mca00 & 34.01 & 20.27 & 154.1 & $1.2 / 1.2$ & 23.16 & 9.2 & 31 & $\mathrm{~J}(\mathrm{~N})$ \\
\hline $\mathrm{J} 1757-2421$ & kom74 & 5.28 & 0.05 & 234.1 & $3.1 / 4.4$ & 4.00 & 3.9 & 29 & $\mathrm{P}(\mathrm{J}, \mathrm{N})$ \\
\hline J1816-0755 & $\mathrm{lfl}+06$ & 21.87 & 4.09 & 217.6 & $3.1 / 2.8$ & 2.48 & 0.17 & 54 & $\mathrm{~J}$ \\
\hline $\mathrm{J} 1841-0524$ & $\mathrm{hfs}+04$ & 27.02 & -0.33 & 445.7 & $4.1 / 5.3$ & 10.42 & 0.2 & 27 & $\mathrm{~J}(\mathrm{P})$ \\
\hline $\mathrm{J} 1853-0004 \dagger$ & $\mathrm{hfs}+04$ & 33.09 & -0.47 & 101.4 & $5.3 / 7.1$ & 21.09 & 0.87 & 43 & $\mathrm{P}(\mathrm{J}, \mathrm{N})$ \\
\hline $\mathrm{J} 1913+1011$ & $\mathrm{mhl}+02$ & 44.48 & -0.17 & 35.9 & $4.6 / 4.8$ & 287.14 & 0.5 & 32 & $\mathrm{~J}$ \\
\hline $\mathrm{J} 1925+1720$ & $\mathrm{lbh}+15$ & 52.18 & 0.59 & 75.7 & $5.1 / 6.9$ & 95.42 & 0.07 & 37 & $\mathrm{~J}$ \\
\hline $\mathrm{J} 1928+1746$ & $\mathrm{cfl}+06$ & 52.93 & 0.11 & 68.7 & $4.3 / 5.8$ & 160.39 & 0.28 & 31 & $\mathrm{~J}(\mathrm{~N})$ \\
\hline $\mathrm{J} 1932+2220 \dagger$ & ht75b & 57.36 & 1.55 & 144.5 & $10.9 / 7.5$ & 75.38 & 1.2 & 44 & $\mathrm{~J}$ \\
\hline $\mathrm{J} 2208+4056 \dagger$ & $\operatorname{slr}+14$ & 92.63 & -12.09 & 637.0 & $1.0 / 0.8$ & 0.080 & 0.48 & 50 & $\mathrm{~J}$ \\
\hline J0636+5129 & $\operatorname{slr}+14$ & 163.91 & 18.64 & 2.86 & $0.2 / 0.5$ & $0.569 \pm 0.001$ & & 48 & $\mathrm{~N}$ \\
\hline $\mathrm{J} 1125-6014 \dagger$ & fsk +04 & 292.50 & 0.89 & 2.63 & $1.0 / 1.5$ & $0.45 \pm 0.3$ & 0.05 & 35 & $\mathrm{P}$ \\
\hline $\mathrm{J} 1327-0755$ & blr +13 & 318.38 & 53.85 & 2.68 & $25.0 / 1.7$ & 0.36 & & 25 & $N(G)$ \\
\hline $\mathrm{J} 1946+3417$ & bck +13 & 69.29 & 4.71 & 3.17 & $7.0 / 5.2(2.5)$ & $0.2 \pm 0.1$ & 0.29 & 29 & $\mathrm{~J}(\mathrm{E})$ \\
\hline
\end{tabular}

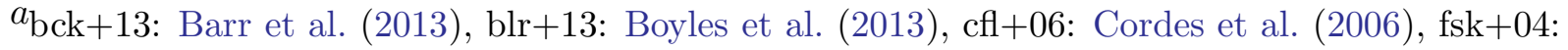

Faulkner et al. (2004), hfs+04: Hobbs et al. (2004a), ht75b: Hulse \& Taylor (1975), kom74: Komesaroff, M. M. 1974, unpublished, lbh+15: Lazarus et al. (2015), lfl+06: Lorimer et al. (2006), lvw68: Large et al. (1968), mca00: McLaughlin et al. (2000), mhl+02: Morris et al. (2002), mlt+78: Manchester et al. (1978), slr+14: Stovall et al. (2014).

while it may be rare for such low-power young pulsars to be seen with the LAT, it is not impossible. We discuss this pulsar further, below.

This new sample allows checks on attempts to understand which pulsars will or will not be seen in gamma rays. Rookyard et al. (2017) focused on the radio profiles of young pulsars with $\dot{E}>10^{35} \mathrm{erg}$ $\mathrm{s}^{-1}$, with the idea that the profiles result from the inclinations of the pulsar's rotation and magnetic axes relative to the line-of-sight $(\zeta$ and $\beta$, respectively). We have seven such pulsars in Table 1 , five of which are in their sample. In their Figure 2, radio pulse width $w_{10}$ versus $\dot{E}$, gamma-ray detected pulsars lie mostly below a diagonal $w_{10}=48 \log \dot{E}-1674$, for $\dot{E}$ in units of erg s ${ }^{-1}$. Our seven 

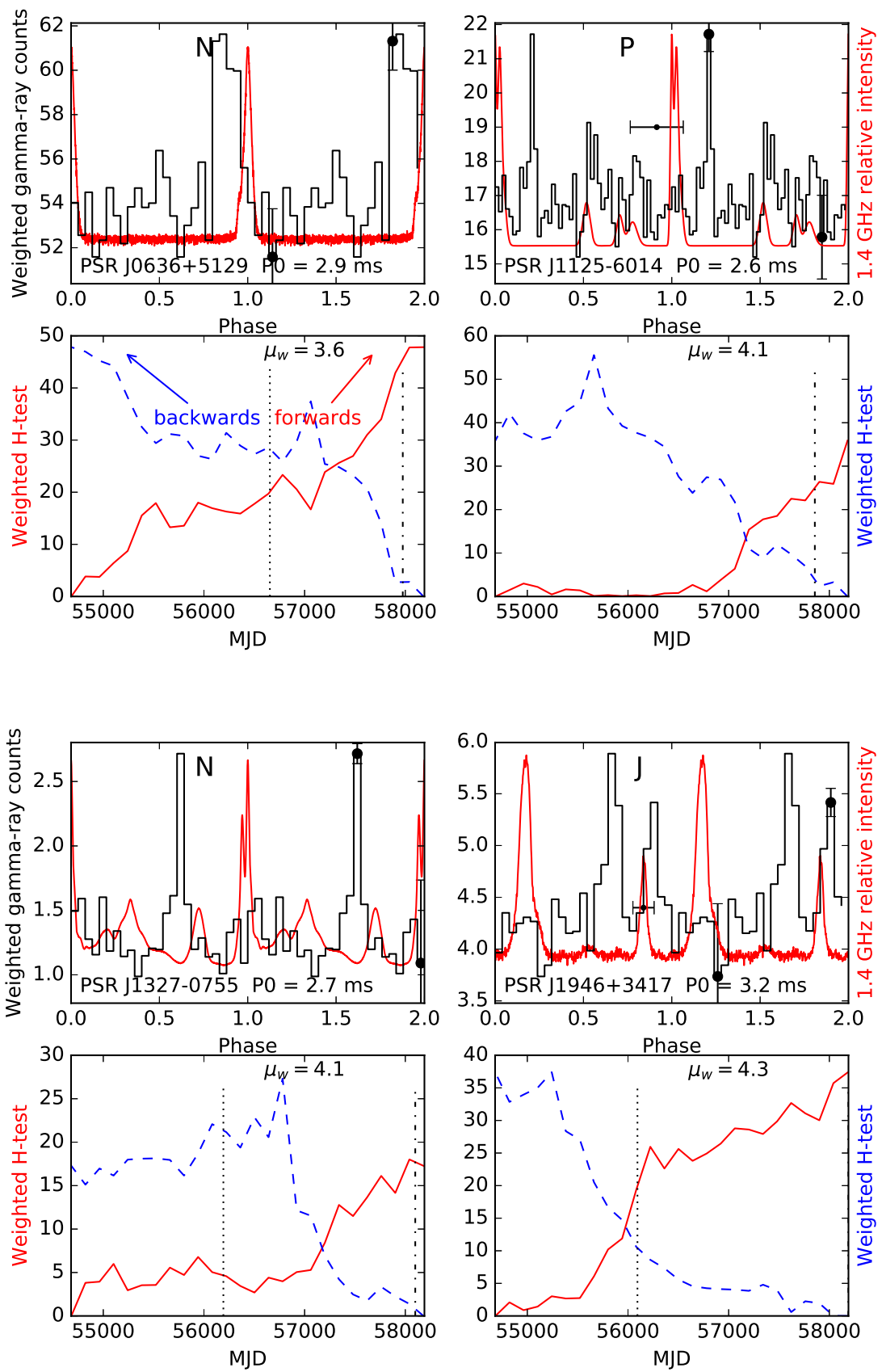

Figure 6. Four MSPs newly discovered in gamma rays. Top: Weighted phase histograms of the $>50$ $\mathrm{MeV}$ gamma rays. The largest and smallest statistical uncertainties are shown. The overlaid $1.4 \mathrm{GHz}$ radio profiles are phase-aligned, with the letter indicating the radio telescope that recorded the profile: N, Nançay; P, Parkes ; J, Jodrell Bank, see the references in Table 1. The DM uncertainty leads to the alignment uncertainty shown by the horizontal error bars for two MSPs. For the other two it is $<0.01$ and is not shown. A full rotation is shown twice, with 25 phase bins (50 bins for J1125-6014). Bottom: H-test significance cumulated starting at both the beginning (solid curve) and at the end (dashed curve) of the dataset. The vertical dotted (dot-dash) line indicates the start (end) of the ephemeris validity. The weighting parameter $\mu_{w}$ used for each pulsar is shown. For PSR J1327-0755 the phase histogram integrates data only after the beginning of the ephemeris validity. For the other pulsars, all data from MJD 54682 until MJD 58182 are used. 
straddle this line. Nevertheless, the general trend they pointed out - that the third of high- $\dot{E}$ pulsars that remain undetected in gamma rays tend to be those with broader radio pulses - is borne out.

For the MSPs, Guillemot et al. (2016) used timing parallax distances, with well-determined uncertainties, to confirm that once relative motion effects have been taken into account via the Shklovskii proper motion corrections, the observed $\dot{E} \approx 10^{33} \mathrm{erg} \mathrm{s}^{-1} \mathrm{MSP}$ deathline value is robust. The four new gamma-ray MSPs reported here are consistent with this.

Again for MSPs, Guillemot \& Tauris (2014) also look for observations that constrain the inclination angles, to then predict whether a gamma-ray beam might sweep the Earth. During the MSP recycling process (i.e., during spin-up), the mass of the precursor of the white dwarf companion determines the orbital radius, and thus the orbital period $P_{B}$. Using $P_{B}$ and $a_{p} \sin i$, they deduce $i$, the orbital inclination. They use $i \approx \zeta$, and expect that gamma rays can be seen mainly for large $\zeta$, since emission models generally favor equatorial gamma-ray beams. Three of our four MSPs have $P_{B}>1$ day, the limit for their model's validity. For two, the $\left(P_{B}, a_{p} \sin i\right)$ values lead to $\zeta$ values near $45^{\circ}$, and for the third $\zeta \approx 70^{\circ}$. These values fill in the $\zeta$ histogram of their Figure 5 , tending to support their conclusions.

\subsection{PSR J2208+4056}

PSR J2208+4056 was originally called J2207+40 when discovered in the Green Bank North Celestial Cap Pulsar Survey (Stovall et al. 2014). An improved rotation ephemeris by Lynch et al. (2018) revealed gamma pulsations directly. Its location well off the Galactic plane, with no other LAT 8-year sources within $1^{\circ} .5$, ensures a low background level and eases detection.

PSR J2208+4056 is the only gamma-ray detection out of 200 pulsars folded in the $10^{32}<\dot{E}<10^{33}$ erg $\mathrm{cm}^{-1}$ decade, for a detection rate of $0.5_{-0.4}^{+0.1 \%}$. Scrutiny of its line-of-sight using the method of Figure 4 in Hou et al. (2014) suggests that its $~ 0.9 \mathrm{kpc}$ DM distance is reliable. Inspection of Figure 7 shows that $<30$ of the folded pulsars are 'nearby', that is, $G_{h}$ is above the detectable minimum.

PSRs J2208+4056 and J1816-0755 are the fifth and sixth radio-interpulse pulsars seen in gamma rays. Radio beams from both magnetic poles of the neutron star sweeping the Earth implies that the pulsars are "orthogonal rotators", that is, that both the magnetic inclination $\alpha=\zeta-\beta$ and the angle $\zeta$ from the line-of-sight to the rotation axes are near 90 (Weltevrede \& Johnston 2008a). We thus observe the neutron star's equator, where the gamma-ray beam is most intense and $f_{\Omega}<1$ (Watters et al. 2009), in addition to seeing both magnetic poles sweep by. Models further predict that equatorial emission is enhanced as pulsars age. With characteristic ages of $\tau_{c}=1.9$ and 0.54 Myr, these two pulsars are among the older ones seen with the LAT. These pulsars' geometry thus favor detection.

Weltevrede \& Johnston (2008b) show that radio polarization in young pulsars is markedly stronger for $\dot{E} \gtrsim 10^{34} \mathrm{erg} \mathrm{s}^{-1}$. Johnston \& Kerr (2018) confirmed the trends using 600 pulsars. It seems an unlikely coincidence that both radio polarization and gamma-ray emission require the same minimum spin parameters for independent reasons. We observed PSR J2208+4056 at $1400 \mathrm{MHz}$ with the Nançay Radio Telescope and obtained a preliminary estimate of $50 \%$ linear polarization. In Figure 8 of Weltevrede \& Johnston (2008b), this places the pulsar among the most polarized for that $\dot{E}$ range. Ideally, an interpulse allows good fits of polarization position angle versus phase, with well-defined $\alpha, \zeta$ values in consequence. Unfortunately, the $1400 \mathrm{MHz}$ flux densities for PSRs J1816-0755 and $\mathrm{J} 2208+4056$ are 170 and $<800 \mu \mathrm{Jy}$, respectively, and the interpulses are $<40 \%$ as intense as the 


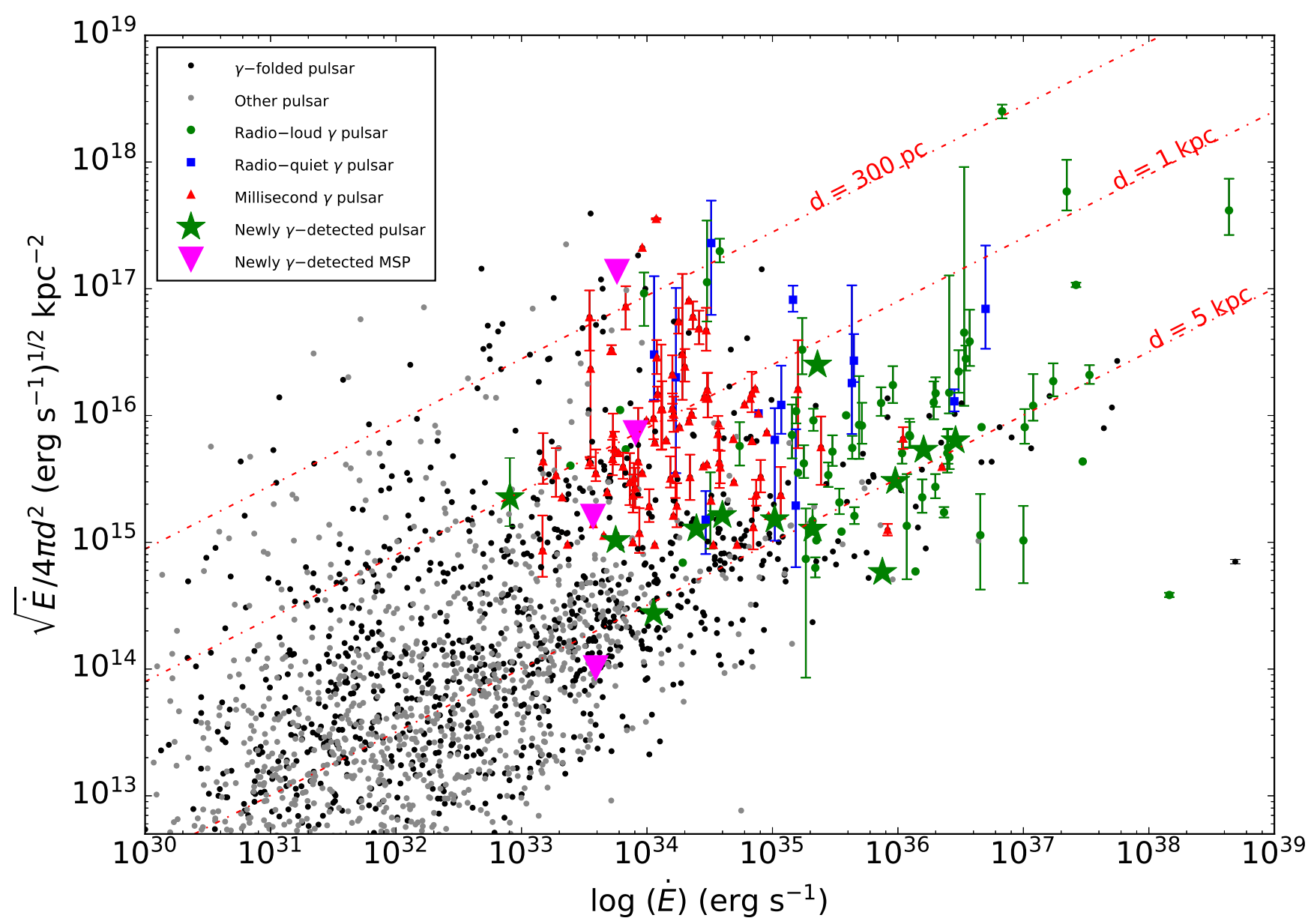

Figure 7. Gamma-ray flux expected for pulsars assuming $L_{\gamma}=\sqrt{10^{33} \dot{E}} \mathrm{erg} \mathrm{s}^{-1}$, for the best distance estimate available, as a function of spindown power $\dot{E}$. The diagonal dashed lines indicate constant distance. Grey dots indicate pulsars not gamma-folded, the other symbol codes are as in Figure 1. Most nearby pulsars with $\dot{E}<10^{33} \mathrm{erg} \mathrm{s}^{-1}$ remain undetected in spite of having heuristic fluxes two orders of magnitude larger than the measured fluxes of many pulsars with higher $\dot{E}$, however the observed gamma-ray emission "deathline" may still be an artifact of selection bias due to the pulsar's distance. For PSR J1327-0755 the second distance in Table 1 is used, instead of first as for the others. To convert the figure's scale to the units of $G_{100}$, multiply by $3.33 \times 10^{-27} \mathrm{erg}^{1 / 2} \mathrm{~s}^{-1 / 2} \mathrm{kpc}^{2} \mathrm{~cm}^{-2}$, see also $2 \mathrm{PC}$ Eq. 18 .

main pulses. Position angle fits to both pulses for either pulsar will be a challenge. Observations at lower frequencies are of interest.

In summary, PSR J2208+4056 establishes that the minimum spindown power for gamma-ray emission is lower than previously observed. Since $L_{\gamma}$ for low-power pulsars is low, detection requires favorable geometry and sky location, partially explaining the low $(\sim 0.5 \%)$ detection rate.

\subsection{Distance Biases}

With our large sample of pulsars we can explore whether the apparent deathline is an artifact of a distance-related selection bias. Figure 7 shows $G_{h}$ versus the spindown power $\dot{E}$, for our twelve 
hundred pulsars. $G_{h}$ is our best predictor of gamma-ray detectability since, as shown in 2PC Figure 9, gamma-ray luminosity scales roughly as $L_{\gamma} \propto \sqrt{\dot{E}}$. Arons (1996) predicted the correlation, arguing that the open field-line voltage is $V \simeq 3.18 \times 10^{-3} \sqrt{\dot{E}}$ volts, and that above some threshold voltage, the size of the gamma-ray emitting electron-positron cascades increases linearly with $V$.

Except for some outliers, the minimum $G_{h}$ for known gamma-ray pulsars in Figure 7 is $G_{h}^{\text {min }}=$ $10^{15}\left(\mathrm{erg} \mathrm{s}^{-1}\right)^{1 / 2} \mathrm{kpc}^{-2}=3.3 \times 10^{-12} \mathrm{erg} \mathrm{cm}^{-2} \mathrm{~s}^{-1}$. The figure seems to show that if pulsars with $\dot{E}<10^{33} \mathrm{erg} \mathrm{s}^{-1}$ emit gamma rays, then we would have detected some of those with high $G_{h}$. However, Figure 3 of Laffon et al. (2015) shows the fraction of folded pulsars detected in gamma rays, as a function of $\dot{E}$. Updating the curves with the recent discoveries preserves the overall trends. For young pulsars, the fraction is $\sim 60 \%$ for $\dot{E}>10^{35} \mathrm{erg} \mathrm{s}^{-1}$, dropping to $\sim 5 \%$ for the decade $10^{33}<\dot{E}<10^{34} \mathrm{erg} \mathrm{s}^{-1}$. With the detection of PSR J2208+4056, we now have $0.5 \%$ detected in the decade below that. For MSPs, $40 \%$ of the pulsars in the low decade are seen, rising to $>80 \%$ for high $\dot{E}$. Hence, the detection efficiency at low $\dot{E}$ is small but non-zero.

Below $\dot{E}<10^{33} \mathrm{erg} \mathrm{s}^{-1}$ Figure 7 shows 48 (46) pulsars with $G_{h}>G_{h}^{\text {min }}$ that we have (not) phasefolded. Over $70 \%$, folded or not, are off the plane, $|b|>5^{\circ}$, where the diffuse background is low. The low efficiency, applied to the small sample, predicts effectively no detections. Low-power pulsars thus may emit gamma rays, but there are not enough within distances accessible by the Fermi LAT for us to know. It will be interesting to add a population of old pulsars to a population synthesis to see whether the low flux per pulsar combined with the large population of low power pulsars adds a noticeable contribution to the diffuse Galactic emission.

\subsection{Spectra}

We evoked, above, cascades of electrons and positrons accelerated by high voltages in the regions where the magnetic field lines open as they cross the light cylinder. The maximum gamma-ray energies from curvature radiation are higher when the field-line radii of curvature are smaller. These radii decrease with rotation period, because the light cylinder is smaller, and when the magnetic inclination $\alpha$ is large. A stronger magnetic field induces higher $V$ for a given period, and thus bigger cascades and, as long as the resulting plasma has not shorted out the electric field, higher $L_{\gamma}$. In this picture, the gamma-ray spectral shape - intensity, hardness (spectral index $\Gamma$ ), and cutoff energy $E_{\text {cut }}$ - depend on the spindown parameters. Modelers aim to refine and quantify this picture.

In particular, Figure 2 of Kalapotharakos et al. (2017) builds upon an empirical result from 2PC, that $\log E_{\text {cut }}$ is a parabolic function of $\log \dot{E}$. Figure 7 in $2 \mathrm{PC}$ shows $\Gamma$ increasing linearly with $\dot{E}$, and we discussed $L_{\gamma} \propto \sqrt{\dot{E}}$ above. Kalapotharakos et al. (2017) suggest that combining the three correlations causes the energy of greatest flux to scale with $\dot{E}$. The deathline would be due not to the emission mechanism abruptly shutting off below some minimum $\dot{E}$, but rather to a combination of reduced intensity and, importantly, emission shifting to the $\mathrm{MeV}$ range where the LAT is less sensitive. As a counterpoint, we mention that the MeV pulsars in Kuiper \& Hermsen (2015) mostly have very high $\dot{E}$. The upcoming Fermi Third Pulsar Catalog will double the number of measured spectra as compared to $2 \mathrm{PC}$, allowing better constraints on how spectral shapes depend on spin parameters.

\section{SUMMARY}

In its $11^{\text {th }}$ year on orbit, Fermi LAT continues to discover about 24 gamma-ray pulsars per year. Two discovery channels are deep radio searches and blind gamma-ray periodicity searches at the 
positions of bright pulsar-like unidentified gamma-ray sources. This paper concerns a third channel, phase-folding gamma rays using a rotation ephemeris obtained from sustained observations of previously known pulsars. This channel is the most sensitive to gamma-faint pulsars.

Gamma-ray pulsations for 16 pulsars were presented, 12 young and 4 recycled, made possible because i) several hundred updated (1269 in total) ephemerides were applied to a 9.6 year gammaray data set ; ii) Bruel (2018)'s gamma-ray weighting technique can be applied regardless of whether or not the pulsar is seen as a phase-integrated point source ; iii) we demonstrated that the detection threshold can be lowered to $4.1 \sigma$ without incurring false positive detections. The new gammaray pulsars include the faintest yet seen, giving fewer than ten photons per hundred days. We described some of the consequences of the resulting large Poisson fluctuations. PSR J2208+4056 has $\dot{E}=8 \times 10^{32} \mathrm{erg} \mathrm{s}^{-1}$, three times lower than what had been the lowest spindown powers for known gamma-ray pulsars these last several years.

We discussed selection biases associated with the new discoveries. Most of the new pulsars have very narrow peaks, improving sensitivity, and lie at low Galactic latitude, where the background is intense. The Third Gamma-ray Pulsar Catalog ("3PC", in preparation) will contain more than twice as many gamma-ray pulsars as $2 \mathrm{PC}$. The unambiguous identification and localisation of gamma-ray pulsars helps improve 4FGL, the upcoming $4^{\text {th }}$ Fermi LAT catalog of sources using 8 years of data. Both catalogs should go to press in the coming year.

Emission modelers should bear in mind that since faint pulsars are easier to detect if the pulses are narrow, tuning models to match the observed sample would be a mistake. If, for example, pulses broaden as $\dot{E}$ decreases, those pulsars would be absent from the current observed sample.

We discussed whether the rarity of detected gamma-ray pulsars with spindown power $\dot{E}<$ $10^{33} \mathrm{erg} \mathrm{s}^{-1}$ is a feature of the gamma-ray emission mechanism (a "deathline"), or is due to observational selection effects. There does seem to be a deathline, with luminosity dropping below the $L_{\gamma} \propto \sqrt{\dot{E}}$ trend at low $\dot{E}$. But faint emission at low $\dot{E}$ for rare pulsars may be undetected at present because the distances implied by the large volumes required to obtain a pulsar sample large enough to provide a few detections impose values of $G_{h}=\sqrt{\dot{E}} /\left(4 \pi d^{2}\right)$ below the LAT's sensitivity. Sensitivity is enhanced for narrow pulses and for high Galactic latitudes, but requiring those conditions decreases the sample size. We also mentioned model predictions that peak emission would shift from the $\mathrm{GeV}$ to the $\mathrm{MeV}$ energy range as $\dot{E}$ decreases, making LAT detections more difficult.

Along with the simple scheme applied here, Bruel (2018) presents a pulsar weighting method that is more sensitive but also more difficult to use. Future work will be to re-fold the thousand pulsars, as well as other pulsars for which ephemerides may become available, with the refined method. Preliminary indications are that we will find still more faint gamma-ray pulsars.

\section{Acknowledgements}

The Nançay Radio Observatory is operated by the Paris Observatory, associated with the French Centre National de la Recherche Scientifique (CNRS).

The Parkes radio telescope is part of the Australia Telescope which is funded by the Commonwealth Government for operation as a National Facility managed by CSIRO.

The Lovell Telescope is owned and operated by the University of Manchester as part of the Jodrell Bank Centre for Astrophysics with support from the Science and Technology Facilities Council of the United Kingdom. 
The Robert C. Byrd Green Bank Telescope (GBT) is operated by the National Radio Astronomy Observatory, a facility of the National Science Foundation operated under cooperative agreement by Associated Universities, Inc.

The Fermi LAT Collaboration acknowledges generous ongoing support from a number of agencies and institutes that have supported both the development and the operation of the LAT as well as scientific data analysis. These include the National Aeronautics and Space Administration and the Department of Energy in the United States, the Commissariat à l'Energie Atomique and the Centre National de la Recherche Scientifique / Institut National de Physique Nucléaire et de Physique des Particules in France, the Agenzia Spaziale Italiana and the Istituto Nazionale di Fisica Nucleare in Italy, the Ministry of Education, Culture, Sports, Science and Technology (MEXT), High Energy Accelerator Research Organization (KEK) and Japan Aerospace Exploration Agency (JAXA) in Japan, and the K. A. Wallenberg Foundation, the Swedish Research Council and the Swedish National Space Board in Sweden.

Additional support for science analysis during the operations phase is gratefully acknowledged from the Istituto Nazionale di Astrofisica in Italy and the Centre National d'Études Spatiales in France. This work performed in part under DOE Contract DE- AC02-76SF00515. Work at NRL is supported by NASA.

\section{REFERENCES}

Abdo, A. A., Ackermann, M., Ajello, M., et al. 2010, ApJ, 714, 927, (PSR B1509-58)

Abdo, A. A., Ajello, M., Allafort, A., et al. 2013, ApJS, 208, 17, (2 ${ }^{\text {nd }}$ Fermi Pulsar Catalog, 2PC)

Acero, F., Ackermann, M., Ajello, M., et al. 2013, ApJ, 773, 77, (LAT PWNe)

—. 2015, ApJS, 218, 23, $\left(3^{\text {rd }}\right.$ Fermi LAT Source Catalog, 3FGL)

-. 2016, ApJS, 224, 8, (SNR catalog)

Ackermann, M., Ajello, M., Baldini, L., et al. 2017, ApJ, 843, 139

Arons, J. 1996, A\&AS, 120, C49

Atwood, W., Albert, A., Baldini, L., et al. 2013, in eConf C121028, 8, Proc. $4^{\text {th }}$ Fermi Symposium, ed. T.J. Brandt, N. Omodei, \&

C. Wilson-Hodge, arXiv:1303.3514

Atwood, W. B., Abdo, A. A., Ackermann, M., et al. 2009, ApJ, 697, 1071, (LAT Instrument Paper)

Barr, E. D., Freire, P. C. C., Kramer, M., et al. 2017, MNRAS, 465, 1711

Barr, E. D., Champion, D. J., Kramer, M., et al. 2013, MNRAS, 435, 2234, (bck+13)

Boyles, J., Lynch, R. S., Ransom, S. M., et al. 2013, ApJ, 763, 80, (blr+13)

Bruel, P. 2018, A\&A, (submitted)
Cameron, A. D., Champion, D. J., Kramer, M., et al. 2018, MNRAS, 475, L57

Carlberg, R. G., \& Innanen, K. A. 1987, AJ, 94, 666

Clark, C. J., Wu, J., Pletsch, H. J., et al. 2017, ApJ, 834, 106

Cognard, I., Guillemot, L., Johnson, T. J., et al. 2011, ApJ, 732, 47

Cordes, J. M., \& Lazio, T. J. W. 2002, arXiv:astro-ph/0207156

Cordes, J. M., Freire, P. C. C., Lorimer, D. R., et al. 2006, ApJ, 637, 446, (cfl+06)

de Jager, O. C., \& Büsching, I. 2010, A\&A, 517, L9

Deneva, J. S., Ray, P. S., Camilo, F., et al. 2016, ApJ, 823, 105

Di Mauro, M., Charles, E., \& Wood, M. 2017, arXiv:1705.00009, submitted to ApJ

Faulkner, A. J., Stairs, I. H., Kramer, M., et al. 2004, MNRAS, 355, 147, (fsk+04)

Frail, D. A., \& Weisberg, J. M. 1990, AJ, 100, 743

Guillemot, L., \& Tauris, T. M. 2014, MNRAS, 439, 2033

Guillemot, L., Smith, D. A., Laffon, H., et al. 2016, A\&A, 587, A109

Hobbs, G., Faulkner, A., Stairs, I. H., et al. 2004a, MNRAS, 352, 1439, (hfs+04) 
Hobbs, G. B., Edwards, R. T., \& Manchester, R. N. 2006, MNRAS, 369, 655

Hobbs, G. B., Lyne, A. G., Kramer, M., Martin, C. E., \& Jordan, C. 2004b, MNRAS, 353, 1311

Hou, X., Smith, D. A., Guillemot, L., et al. 2014, A\&A, 570, A44

Hulse, R. A., \& Taylor, J. H. 1975, ApJL, 201, L55, (ht75b)

Johnston, S., \& Kerr, M. 2018, MNRAS, 474, 4629

Kalapotharakos, C., Harding, A. K., Kazanas, D., \& Brambilla, G. 2017, ApJ, 842, 80

Kaplan, D. L., Escoffier, R. P., Lacasse, R. J., et al. 2005, PASP, 117, 643

Kerr, M. 2011, ApJ, 732, 38

King, I. 1962, AJ, 67, 471

Kuijken, K., \& Gilmore, G. 1989, MNRAS, 239, 571

Kuiper, L., \& Hermsen, W. 2015, MNRAS, 449, 3827

Laffon, H., Smith, D. A., \& Guillemot, L. 2015, Proc. $5^{\text {th }}$ Fermi Symposium - eConf C141020.1, arXiv:1502.03251

Large, M. I., Vaughan, A. E., \& Wielebinski, R. 1968, Nature, 220, 753, (lvw68)

Lazarus, P., Brazier, A., Hessels, J. W. T., et al. 2015, ApJ, 812, 81, (lbh+15)

Lorimer, D. R., Faulkner, A. J., Lyne, A. G., et al. 2006, MNRAS, 372, 777, (lfl+06)

Lynch, R. S., Swiggum, J. K., Kondratiev, V. I., et al. 2018, ApJ, 859, 93, (lsk+18)

Manchester, R. N., Hobbs, G. B., Teoh, A., \& Hobbs, M. 2005, AJ, 129, 1993

Manchester, R. N., Lyne, A. G., Taylor, J. H., et al. 1978, MNRAS, 185, 409, (mlt+78)

McLaughlin, M., Cordes, J. M., \& Arzoumanian, Z. 2000, in Astronomical Society of the Pacific Conference Series, Vol. 202, IAU Colloq. 177: Pulsar Astronomy - 2000 and Beyond, ed. M. Kramer, N. Wex, \& R. Wielebinski, 41, (mca00)
Moffat, A. F. J. 1969, A\&A, 3, 455

Morris, D. J., Hobbs, G., Lyne, A. G., et al. 2002, MNRAS, 335, 275, (mhl+02)

Ng, C., Champion, D. J., Bailes, M., et al. 2015, MNRAS, 450, 2922

Pleunis, Z., Bassa, C. G., Hessels, J. W. T., et al. 2017, ApJL, 846, L19

Ray, P. S., Kerr, M., Parent, D., et al. 2011, ApJS, 194, 17

Read, A. M., Rosen, S. R., Saxton, R. D., \& Ramirez, J. 2011, A\&A, 534, A34

Romani, R. W., Kerr, M., Craig, H. A., et al. 2011, ApJ, 738, 114

Rookyard, S. C., Weltevrede, P., Johnston, S., \& Kerr, M. 2017, MNRAS, 464, 2018

Shannon, R. M., \& Cordes, J. M. 2010, ApJ, 725, 1607

Shklovskii, I. S. 1970, Soviet Astronomy, 13, 562

Shternin, P. S., Yu, M., Kirichenko, A. Y., et al. 2017, Journal of Physics: Conference Series, 932, 012004

Smith, D. A., \& Thompson, D. J. 2009, in Astrophysics and Space Science Library, Vol. 357, Astrophysics and Space Science Library, ed. W. Becker, 621

Smith, D. A., Guillemot, L., Camilo, F., et al. 2008, A\&A, 492, 923

Stovall, K., Lynch, R. S., Ransom, S. M., et al. 2014, ApJ, 791, 67, (slr+14)

Verbiest, J. P. W., Weisberg, J. M., Chael, A. A., Lee, K. J., \& Lorimer, D. R. 2012, ApJ, 755, 39

Watters, K. P., Romani, R. W., Weltevrede, P., \& Johnston, S. 2009, ApJ, 695, 1289

Weltevrede, P., \& Johnston, S. 2008a, MNRAS, 387,1755

—. 2008b, MNRAS, 391, 1210

Weltevrede, P., Johnston, S., Manchester, R. N., et al. 2010, PASA, 27, 64

Yao, J. M., Manchester, R. N., \& Wang, N. 2017, ApJ, 835, 29 